Trends Endocrinol Metab. 2014 September ; 25(9): 472-480. doi:10.1016/j.tem.2014.05.003.

\title{
The role of BMPs in endothelial cell function and dysfunction
}

\author{
Laura A. Dyer ${ }^{1}$, Xinchun $\mathbf{P i}^{1}$, and Cam Patterson ${ }^{2}$ \\ ${ }^{1}$ McAllister Heart Institute, University of North Carolina at Chapel Hill, Chapel Hill, NC 27599 \\ ${ }^{2}$ New York-Presbyterian Hospital/Weill-Cornell Medical Center, New York, NY 10065
}

\begin{abstract}
The bone morphogenetic protein (BMP) family of proteins has a multitude of roles throughout the body. In embryonic development, BMPs promote endothelial specification and subsequent venous differentiation. The BMP pathway also plays important roles in the adult vascular endothelium, promoting angiogenesis and mediating shear and oxidative stress. The canonical BMP pathway functions through the Smad transcription factors; however, other intracellular signaling cascades can be activated, and receptor complexes beyond the traditional type I and type II receptors add additional layers of regulation. Dysregulated BMP signaling has been linked to vascular diseases, including pulmonary hypertension and atherosclerosis. This review addresses recent advances in the roles of BMP signaling in the endothelium and how BMPs affect endothelial dysfunction and human disease.
\end{abstract}

\section{BMPs in endothelial cells}

The importance of the BMP (see Glossary) pathway in vascular development has been known for years. Beyond its importance in embryonic development, critical roles have been identified in vascular disorders, including hereditary hemorrhagic telangiectasia (HHT) and peripheral arterial hypertension (PAH) [1]. However, the BMP pathway has functions beyond those in endothelial differentiation, venous specification, and angiogenesis, during development [2]. Recent studies have shown that the BMP pathway also affects processes such as the endothelial response to hypoxia and inflammatory stimuli. These additional roles highlight the significance of the BMP pathway in maintaining vascular homeostasis.

Of the numerous BMP ligands and receptors (see $[2,3]$ for detailed reviews and Table 1 for a summary of the ligands and receptors described herein), most of them (BMPs 1, 2, 4, 6, 7, 9, and 10) have shown some effects in endothelial cells. The roles of BMP6 and BMP7 are becoming better understood, and their contributions to human diseases such as cerebral cavernous malformation (CCM) make these ligands crucial to study further (e.g., [4-6]). However, this review will focus on BMPs 2, 4, and 9 due to their welldefined roles in the

() 2014 Elsevier Ltd. All rights reserved.

Corresponding author: Dyer, L.A. (ldyer1@email.unc.edu).

Publisher's Disclaimer: This is a PDF file of an unedited manuscript that has been accepted for publication. As a service to our customers we are providing this early version of the manuscript. The manuscript will undergo copyediting, typesetting, and review of the resulting proof before it is published in its final citable form. Please note that during the production process errors may be discovered which could affect the content, and all legal disclaimers that apply to the journal pertain. 
vascular endothelium and recent studies that are addressing how these specific BMP signaling cascades affect endothelial dysfunction and human disease.

\section{BMP2 and BMP4}

Of the BMPs, BMP2 and BMP4 are best characterized. These ligands typically associate with type I receptors BMPR1a (Alk3) or BMPR1b (Alk6) and BMPRII, leading to the phosphorylation of Smads 1, 5, and 8 (Smad1/5/8) (Figure 1A, B; reviewed in [7]). BMP2 and BMP4 share considerable sequence homology and many functions. In bovine aortic endothelial cells (BAECs), BMP2 and BMP4 can increase proliferation and tube formation [8]. This effect can be inhibited by the binding of matrix Gla protein (MGP) [8], which is enriched in the lungs and kidney; knocking out MGP increases BMP4-induced vascular endothelial growth factor (VEGF) signaling, leading to increased lung endothelial cell proliferation [9].

\section{BMP9}

Circulating factor BMP9 interacts with endothelially restricted Alk1 (ACVRL1), a type I serine/threonine-protein kinase and cell-surface receptor for the TGF-beta superfamily of ligands, as well as BMPRI [10-16]. In mouse embryonic stem cell-derived endothelial cells, the BMP9-Alk1 interaction induces the expression of VEGFR2 and the angiopoietin receptor, Tie2, leading to increased proliferation and tube formation [10]. Similar effects are observed in in vivo models such as Matrigel plugs and tumor xenografts [10]. (See Text Box 1 for more detail on the in vivo models described herein.) Human umbilical vein endothelial cells (HUVECs) show increased tube formation in response to BMP9 treatment [17], and human pulmonary artery endothelial cells (HPAECs) show increased tube formation in response to BMP9-induced endothelin-1 expression [18]. Although Smad4 generally acts as a co-factor for all Smads, BMP9's effect via endothelin-1 is Smad4-independent [18]. In contrast with the effect of BMP9 in HUVECs and HPAECs, the BMP9-Alk1 interaction inhibits proliferation and migration in other endothelial populations such as human dermal microvascular endothelial cells and human aortic endothelial cells (HAECs) [7, 19]. These differing roles suggest that additional factors determine whether BMP9 promotes or inhibits these cellular behaviors and highlight the complexity of even a single BMP ligand.

\section{BMP receptors}

Endothelial cells express different combinations of the type I and type II BMP receptors. For example, human microvascular endothelial cells, HUVECs, rat and mouse aortic endothelial cells, and mouse endothelial cells that are responsive to BMP2/4, typically express either BMPR1a or BMPR1b and BMPRII and respond to the BMP ligand by inducing Smad1/5/8 phosphorylation [20-24]. In some cases, BMPR1b associates with activin A type II receptor (ActRIIa) [25], and BMP2-induced activation of ActRIIa leads to Smad3 activation [26]. In contrast to BMP2/4-responsive cells, BMP9-responsive cells (e.g., mouse embryonic stem cell-derived endothelial cells, murine embryonic endothelial cells, and human dermal microvascular endothelial cells) express Alk1 and BMPRII [10, 13, 27]. However, Alk1 may also associate with ActRIIA and B, which can lead to the phosphorylation of either the canonical Smad1/5 [13, 15] or Smad2 [19]. 
In addition to the type I and II receptors, a new set of co-receptors, termed the type III receptors, are being identified and characterized (Figure 1C). Endoglin is an endothelially expressed type I glycoprotein that mediates crosstalk between integrins and the BMP family [27]. In murine embryonic endothelial cells, fibronectin and integrin a $5 \beta 1$ promote endoglin's association with Alk1, leading to internalization via endocytosis and increased Smad1/5 phosphorylation via BMP9 or TGF- $\beta 1$ [27].

Additionally, low-density lipoprotein receptor-related protein 1 (LRP1) acts as a co-receptor for BMPR1b and competes with BMPRII to bind to BMPR1b [22]. LRP1 directs the BMPreceptor complex to the endocytic recycling pathway, which recycles the BMP ligandreceptor complex back to the cell membrane, thus concentrating the BMP ligand-receptor complex and increasing its downstream signaling [22]. Based on the numerous intracellular cascades that can be affected via BMPs, additional co-receptors that lead to specific cascades are anticipated.

\section{BMP regulators}

The BMP pathway comprises a diverse set of ligands, receptors, and intracellular signaling molecules. An additional level of regulation is afforded through its numerous agonists and antagonists. The most recently identified BMP regulator is BMP-binding endothelial regulator (BMPER), which both promotes and inhibits signaling via BMP4, depending on the context [28]. For example, BMPER-haploinsufficient mice show increased Smad1/5/8 phosphorylation in the greater curvature of the aortic arch and in retinal endothelial cells compared with wild-type mice, indicating that BMPER represses canonical signaling in these contexts [29, 30]. However, when BMP4 and BMPER associate with the LRP1/ BMPR1b dimer, BMP4 is internalized via endocytosis. As discussed above, this internalization leads to increased Smad1/5/8 phosphorylation and explains the pro-BMP effects of BMPER [22]. Consistent with this pro-BMP outcome, zebrafish treated with a BMPER morpholino show a dorsalized phenotype and mispatterned intersegmental vessels, indicating that BMPER acts as a BMP agonist during zebrafish development [31].

Although well-defined roles exist for BMP2/4 and BMP9, additional roles are continually uncovered for additional BMP ligands. In combination with the numerous ligand-receptor complexes and regulators, this pathway offers complex ways of regulating endothelial cell behavior (e.g., Figure 1D-F). Thus, understanding the different components of the pathway and how they interact will continue to be an important part of determining the effect of BMP signaling in endothelial cells.

\section{The role of BMP signaling during angiogenesis}

BMPs typically promote angiogenesis by increasing and inducing endothelial motility and invasion and by promoting proliferation [2]. HUVECs express the BMP receptors BMPRII and BMPR1b at high levels, and treating HUVECs with exogenous BMP2 increases their motility and invasion [20]. Although BAECs show decreased apoptosis in response to BMP2 treatment [32], HUVECs do not show changes in apoptosis in response to BMP2 [20]. These differential responses may be explained by endothelial heterogeneity. Further, the BMP ligand expression level may also be critical for regulating angiogenesis. In 
transgenic mice overexpressing BMP4 specifically in retinal pigment epithelial cells, Smad1/5 phosphorylation is upregulated and VEGF and matrix metalloprotease-9 expression is reduced compared with wild-type mice; this reduced expression correlates with reduced angiogenesis in a choroidal neovascularization model [33].

Supporting the importance of context in BMP function, the BMP pathway plays a role in arterial versus venous specification. Within the caudal vein plexus, BMP2 and the BMP receptors BMPR2a and BMPR2b are restricted to the developing cardinal vein, and axial veins sprout in response to BMP2 [34]. This effect occurs via the R-Smads (i.e., Smad1/5/8 and Smad2/3), which promote venous sprouting, and the extracellular signal-regulated kinases (ERKs), which promote venous sprout length [34]. Further, the clathrin-associated sorting pathway protein disabled homolog 2 (Dab2) mediates angiogenesis in veins but not arteries [35]. Dab2 is also restricted to venous cells in the developing zebrafish and promotes the internalization of BMPRII via the clathrincoated pit pathway [35]. Like the LRP1/BMPR1b-mediated internalization of BMP4 [22], the Dab2-mediated endocytosis of BMPRII promotes downstream Smad1/5/8 phosphorylation [35].

Unlike BMP2/4, circulating BMP9 appears to have a different role. Upon activation, platelets release BMP9, which inhibits lymphatic endothelial cell migration but has no effect on HUVECs [36]. Interestingly, blocking circulating BMP9 increases retinal vascularization in postnatal wild-type mouse pups, suggesting that BMP9 inhibits endothelial migration in this context $[37,38]$. A similar phenotype is observed by blocking the main receptor for BMP9, Alk1, via adenovirus [38]. Intriguingly, global BMP9-deficient mice show no gross defects due to the compensatory effect of BMP10 [37]. Inhibiting BMP9 in wild-type pups with a neutralizing antibody leads to increased retinal vascular density, which is not observed in the BMP9-deficient mouse and suggests that the antibody is cross-reactive. However, if BMP10 signaling is also blocked in BMP9-deficient mouse pups by administering a BMP10-neutralizing antibody, retinal expansion is blocked; in contrast, this antibody has no effect on retinal expansion in wild-type pups, further supporting the redundancy of BMP9 and BMP10 [37].

BMP9 and BMP10 bind to Alk1, which induces mRNA expression of the ALK1 receptor signaling-dependent gene, transmembrane protein 100 (Tmem100), within the arteries [39]. The Tmem100-deficient mouse displays disorganized arteries and downregulated Notch/Akt signaling [39]. Additionally, Alk1-mediated BMP9 signaling leads to the induction of Notch pathway components Hey1 and Hey2, which repress tube formation in HUVECs [38]. Conditionally knocking out Smad1/5 in the endothelium further leads to the downregulation of Notch pathway members, such as Hey1, Hes1, and Jagged1, and the inhibition of sprouting in the hindbrain of E9.5 embryos [40]. Unfortunately, the early embryonic lethality of these embryos precludes studying other aspects of vascular development [40]. Together, these defects are consistent with the role of the Notch pathway in repressing sprouting and promoting arterial development [41] and indicate that BMP9/10 can inhibit endothelial tube elongation by promoting the Notch pathway.

BMP9 also induces BMPER mRNA and protein expression in HAECs via its interaction with Alk1, which feeds back to inhibit BMP9/Alk1-induced signaling [42]. BMPER 
preferentially binds to BMP9 over BMP4; thus, the BMPER-BMP9 interaction allows BMP4/Alk2 (also known as ACVR1) signaling to remain unaltered [42]. BMPER inhibits VEGF expression in Alk1-expressing HAECs, and the BMPER-deficient embryo shows increased expression of the endothelial marker CD31 in overly thick vessels [42]. However, the significance of this thickened endothelium requires further investigation.

Although the BMP family is often considered pro-angiogeneic, these experiments highlight how context affects whether a BMP ligand is pro- or anti-angiogenic. These differences may be due to the differential expression of co-receptors that mediate BMP internalization, which may help explain why different endothelial cell types exhibit varying responses to the same BMP ligands. Additional studies, particularly into coreceptors, should help clarify the apparently contradictory roles of BMPs in angiogenesis.

\section{The interaction between BMP signaling and oxygen in angiogenesis}

Hypoxia is a major inducer of angiogenesis, and the BMP pathway mediates the endothelial response to low oxygen. BMP9 downregulates apelin via BMPRII and the R-Smad intracellular pathways in microvascular endothelial cells exposed to hypoxia [43]. This downregulation blocks apelin-induced endothelial proliferation and allows hypoxia-induced angiogenesis [43]. Upstream of the BMP ligands, BMPER is downregulated in response to hypoxia, which relieves the BMPER-induced downregulation of BMP signaling via both the Smad and ERK1/2 pathways [30]. In an oxygen-induced retinopathy mouse model, the induced hypoxia leads to both decreased BMPER expression and increased vascularization [30], supporting the role of BMPER in inhibiting angiogenesis. Additionally, both in vitro (in HAECs) and induced myocardial infarction mouse models show that hypoxia induces the endothelial expression of Alk1, phosphorylated Smad1/5, and endoglin [44]. Endoglin then leads to increased BMP-response element activity and expression of Inhibitor of differentiation (ID)1 and BCL-X (B-cell CLL/lymphoma-X, also known as BCL2-like 1), which increase HAEC proliferation and may thus promote angiogenesis [44]. Further supporting the protective, pro-angiogenic role of the BMP pathway, treating mice with the BMP antagonist BMP and activin membrane-bound inhibitor (BAMBI) inhibits neovascularization in a femoral arterial denudation injury model [45].

In addition to the effect of reduced levels of oxygen, oxidative stress can also affect vascular function. Endothelial cells endogenously produce reactive oxygen species (ROS) through mitochondrial electron transport chain reactions and nicotinamide adenine dinucleotide phosphate (NADPH) oxidase, and these low levels of ROS generally promote angiogenesis [46]. In contrast, both chronic ROS exposure and exposure to high levels of ROS, due either to the overproduction of ROS or a reduction in ROS scavengers, are detrimental [46]. Arteries that are exposed to disturbed flow show increased BMP4 expression and ROS production, which lead to endothelial dysfunction [23]. BMP4 and ROS stimulate the p38MAP/JNK pathway, leading to caspase 3 activity and apoptosis in aortic endothelial cells from rats and mice [23]. Additionally, treating aortic ring explants with BMP4 induces endothelial contraction at the expense of relaxation and promotes ROS production [24]. By binding to BMPR1A, BMP4 induces ROS production in aortic ring explants, leading to p38 activation and cyclooxygenase 2 (COX2) upregulation [24]. Additionally, BMP4-induced 
ROS via BMPR1a induces vasoconstriction in diabetic patients [47]. This constriction can be inhibited in mouse aortic rings by blocking the BMP pathway via the antagonists noggin and mtatin, and noggin reduces ROS levels in the aorta when administered to diabetic mice [47].

As described herein, oxygen serves as a potent regulator of BMP-mediated endothelial cell behavior. In response to hypoxia, BMPs 4 and 9 can promote endothelial proliferation and angiogenesis, which would restore normal oxygen levels. Further, low levels of ROS can promote BMP-mediated angiogenesis. In contrast, oxidative damage via ROS can lead to BMP-mediated endothelial apoptosis and vasoconstriction. Thus, the BMP pathway must be tightly regulated to avoid these pathological conditions.

\section{The BMP pathway in vascular pathologies}

\section{The role of BMP signaling in hypertension}

Hypertension is the leading chronic risk factor for mortality, and despite significant efforts to control the mortality associated with hypertension, the prevalence is increasing in the U.S. [48]. Multiple BMP ligands have well-established roles in pulmonary hypertension, and BMP receptor mutations also affect the response to oxidative stress. BMP2/4 normally stimulate BMPRII to promote the phosphorylation and activity of endothelial nitric oxide synthase (eNOS) via protein kinase A activation, leading to pulmonary artery endothelial cell (PAEC) proliferation, survival, and migration [49]. However, BMP2/4 cannot stimulate PAECs that carry the BMPRII mutations found in patients with pulmonary arterial hypertension (PAH) [49]. These cells exhibit decreased eNOS levels and loss of active NO, which contribute to the PAH phenotype observed in patients with PAH [49].

In response to hypoxia-induced pulmonary hypertension, BMP2 expression increases, leading to increased eNOS expression and activity in intrapulmonary arteries [50]. Lung endothelial cells isolated from BMP2-haploinsufficient mice cannot induce eNOS in response to hypoxia, and BMP2-haploinsufficient mice show more severe pulmonary hypertension than wild-type mice [50]. Additionally, BMP2 mediates endothelial cell survival in PAECs through the canonical Wnt pathway via binding to BMPRII, which induces ERK phosphorylation and GSK3 $\beta$ activation, and through the non-canonical Wnt pathway via binding to ActRIIa, which activates Smad3 and Rho/Rac [26]. BMP2-induced angiogenesis occurs through both Wnt pathways, and impaired angiogenesis contributes to vascular pathologies such as hypertension [26]. However, BMP2 can also induce Smad1 phosphorylation via binding to BMPRII or ActRIIa, leading to increased motility [26].

Despite the high amino acid sequence identity between BMP2/4 and their similar expression patterns, BMP4-haploinsufficient mice are protected from pulmonary hypertension [50]. However, differences in expression levels or cellular localization may account for the severe hypertensive phenotype of the BMP2-haploinsufficient mouse [50]. In addition to the different hypertensive responses induced by the loss of BMP2/4, with loss of BMP2 exacerbating and loss of BMP4 protecting against pulmonary hypertension, excess BMP signaling is also detrimental. Treating spontaneously hypertensive rats, which have up- 
regulated BMP4 expression, with the BMP antagonist Noggin restores endothelial relaxation [24].

BMP9 may also contribute to the pathogenesis of PAH. BMP9 induces endothelin-1 expression in HPAECs via both Smad1 and p38 [18]. This induction inhibits endothelial cell migration while promoting tube formation [18]. Additionally, BMP9 can bind to both Alk1 and BMPRII to stimulate endothelin-1 secretion from the pulmonary microvascular endothelial cells via Smad1/5 and Smad2 phosphorylation, respectively [19, 51]. BMP9induced Smad1/5 phosphorylation leads to Id 1 and Id 2 mRNA expression, whereas BMP9induced Smad2 phosphorylation leads to interleukin- 8 and E-selectin expression and consequently inhibited DNA synthesis in HPAECs [19]. Thus, BMP9-mediated endothelin-1 expression contributes to endothelial stability and may indirectly affect PAH.

The best characterized BMP pathway member in the development of PAH is BMPRII, which is mutated in $10-40 \%$ of patients with idiopathic and $70 \%$ of patients with familial PAH (also known as hereditary PAH) [52]. These mutations lead to loss of function and/or reduced expression of BMPRII [52]. PAECs from patients with these mutations show impaired function, with increased cell death and reduced angiogenesis, leading to decreased integration into the microvasculature when injected into severe immunodeficiency mice [26]. BMPRII also forms a complex with peroxisome proliferator-activated receptor-gamma and $\beta$-catenin, which induces expression of the peptide apelin [53]. As in microvascular endothelial cells [43], apelin promotes endothelial cell survival in PAECs and inhibits pulmonary artery smooth muscle proliferation [53]. Thus, based on the reduction in BMPRII in patients with PAH [52], it is unsurprising that these patients also show diminished levels of apelin [53].

The well-characterized role of BMPRII in PAH has allowed the investigation of pharmacological interventions. The immunosuppressant FK506 (i.e., tacrolimus) binds to the BMP repressor FKBP12, thus relieving FKBP12's repression of the BMP pathway and promoting BMP signaling via Alk1, 2, and 3 [54]. This derepression leads to Smad1/5 and MAPK activity [54]. BMPRII signaling via the canonical Smads and the MAPK pathway can be rescued in PAECs isolated from patients with idiopathic PAH by low-dose treatment with FK506 [54]. Further, administering FK506 to inducible endothelial-specific BMPRIIdeficient mice protects them from developing PAH [54].

\section{The role of BMP signaling in vascular disorders}

One of the clearest examples of a BMP-mediated vascular disorder is HHT. This disorder is caused by abnormal endothelial cell proliferation and smooth muscle recruitment, leading to arteriovenous malformations and leaky vessels [1]. Thus far, three genes (endoglin, Alk1, and Smad4) have been associated with HHT, all of which participate in the BMP pathway [1]. In pulmonary endothelial cells, BMP9-stimulated Alk1 inhibits FGF-induced migration as well as tube formation, suggesting that excess migration may contribute to the vascular anomalies observed in patients with Alk1 mutations [55]. Cross-talk with the Notch pathway has also been implicated, with Smadinduced Notch signaling leading to an inhibition of VEGF and, consequently, inhibited cell sprouting [38]. Although Notch pathway mutations have not been associated with HTT specifically, the Notch ligands Jagged1 and 2 are up- 
regulated in a mouse model of cerebral arteriovenous malformations [56], supporting the importance of this pathway as well. Intriguingly, though, the BMP antagonist MGP also plays a role in titrating the level of BMP signaling [9]. Mice that are deficient for MGP show increased BMP signaling, as expected, but also the arteriovenous malformations that are characteristic of HHT [9]. Together, these results stress the importance of the dose of BMP signaling in promoting normal vascular formation.

\section{The role of BMP signaling in atherosclerosis}

Atherosclerosis is a major problem in the U.S., with prevalence ranging from 35-70\% depending on ethnicity and gender [57]. Myriad factors influence atherosclerosis, and the BMP pathway is involved in many of these processes. One of the major mouse models of atherosclerosis is the ApoE-deficient mouse. ApoE-deficient mice have high expression levels of Hsp70, which binds to MGP and prevent it from inhibiting BMP2/4 [8]. In BAECs, this enhanced BMP signaling leads to increased proliferation and tube formation along with increased calcium secretion, which are consistent with the development of atherosclerotic plaques [8]. Overexpressing MGP reduces canonical BMP signaling, leading to reduced lesions and calcification in ApoE-deficient mice on a high-fat diet [58]. This increased MGP signaling suppresses VEGF, Alk1, and inflammation markers such as intercellular adhesion molecule 1 (ICAM) [58]. In contrast, the phenotype of the MGP-deficient mouse is too severe to cross with the ApoE-deficient mouse and subject to a high-fat diet [58]. The MGPdeficient mouse displays atherosclerosis that is comparable to the ApoE-deficient mouse on a high-fat diet, and MGP-deficient mice display increased BMP signaling, including increased Smad1/5/8 phosphorylation and expression of VEGF and Alk1 [58].

The endothelial dysfunction that underlies atherosclerosis has numerous causes. Certain forms of shear stress lead to inflammation, thickening of the arterial wall, and the formation of cholesterol-filled plaques in the arteries [59]. Importantly, the BMP pathway plays a role in all these steps, as described in the following sections.

Shear stress-induced BMP signaling-Similarly to how ROS can have both positive and negative effects depending on the dose, the shear stress caused by blood flow can also induce different effects. Laminar shear stress, which is present in high-flow arteries that are at low risk of developing atherosclerotic plaques, inhibits BMP4 [60]. In contrast, oscillatory shear stress is found in arteries with disturbed flow, is associated with the development of atherosclerotic plaques, and induces BMP4 expression [61]. Additionally, BMPRII mediates association between BMPR $1 b$ and integrin $a \mathrm{~V} \beta 3$ in endothelial cells in response to oscillatory shear stress [62]. The BMPR1b-integrin aV $\beta 3$ interaction activates the Shc/FAK/ERK pathway, which consequently activates Smad1/5 [62]. This signaling cascade leads to endothelial proliferation, which is one of the earliest steps in atherosclerotic plaque formation, via Runx2, mTOR, and p70S6K [62] and links the BMP pathway to mechanotransductive transmembrane proteins that respond to shear stress [63].

BMPER-haploinsufficiency accelerates atherosclerotic development in ApoE-deficient mice on a high-fat diet [29]. siRNA-mediated knockdown of BMPER in HUVECs leads to increased expression of the inflammatory markers ICAM and vascular cell adhesion 
molecule 1 (VCAM) in response to oscillatory shear stress and "healthy" laminar shear stress [29]. Further supporting its protective role, BMPER promotes eNOS expression in HUVECs under laminar shear stress [29]; eNOS has protective effects in the endothelium, particularly during ischemia and reperfusion [64].

Inflammation and BMP signaling-BMP2 induces inflammation in HUVECs by inducing the expression of ICAM-1 and NF-kB. In HUVECs, TNF- $\alpha$ and NF-kB can downregulate BMPER at the mRNA level during the inflammatory response, which leads to increased expression of inflammation markers ICAM-1 and VCAM-1 and decreased expression of eNOS [65]. A similar downregulation of BMPER mRNA is observed in an LPS-induced inflammation model in mice [65]. In support of BMPER's anti-inflammatory role, BMPER-haploinsufficient mice have reduced eNOS levels and exhibit proinflammatory phenotypes compared with wild-type mice [65].

Pharmacologically, BMP-mediated inflammation can be ameliorated by statins. Mevastatin promotes BMPER expression in HUVECs and C57/B16 mice by inactivating RhoA and stabilizing BMPER mRNA [66]. Thus, inactivating RhoA leads to increased BMPER expression, which inhibits ICAM-1 expression in HUVECs [66]. Additionally, mevastatin downregulates BMP4 at the mRNA and protein level in HUVECs [66], further increasing the BMPER:BMP ratio that protects blood vessels from inflammation and highlighting the dose-dependent context through which BMPER serves as a pro- or anti-BMP regulator [28].

In addition to BMPRII's role in PAH, BMPRII has an anti-inflammatory function. Proatherogenic flow (i.e., oscillatory shear stress) inhibits BMPRII expression, whereas antiatherogenic flow (i.e., laminar shear stress) promotes BMPRII expression [67]. BMPRII expression is downregulated in patients with coronary artery disease with atherosclerotic plaques [67]. BMPRII-haploinsufficiency accelerates atherosclerosis in ApoE-deficient mice, even when the mice are maintained on a normal diet [67]. In HUVECs, BMPRII knockdown inhibits BMP signaling through Smad1/5/8, thus increasing ICAM expression and leukocyte adhesion [67]. BMPRII knockdown also results in increased ROS production and Nox1 expression via NF-kB [67]. Of the BMP receptors, BMPRII is the only known receptor to protect against inflammation [67].

The effect of cholesterol on BMP signaling-In addition to being upregulated by proatherogenic flow and inflammation, BMP2 expression is induced by oxidized lowdensity lipoprotein (LDL) in coronary artery endothelial cells and human arterial endothelial cells $[68,69]$. In human arterial endothelial cells, BMP2 is the only BMP ligand to be upregulated at the mRNA level by oxidized LDL [68]. In coronary artery endothelial cells, this induction occurs through toll-like receptors 2 and 4, which stimulate the proinflammatory NF-kB and ERK pathways [69]. Intriguingly, oxidized LDL does not stimulate inflammatory cytokine production in these cells, suggesting that NF-kB and ERK instead promote coronary artery calcification, which exacerbates plaque formation [69].

The small molecule LDN-193189 inhibits BMP signaling and leads to downregulated ROS production via oxidized LDL [68]. When administered to either LDL receptor (LDLR)deficient mice or wild-type mice on a high-fat diet, LDN-193189 decreases LDL synthesis 
and protects against hepatic steatosis (i.e., inflammation of the liver) [68]. This small molecule also inhibits lesion formation and arterial calcification in the LDLR-deficient mice independently of changes in body size. Because obesity can cause lesion formation and arterial calcification, this lack of change in size in the treated LDLR-deficient mice precludes improved health as the cause of these ameliorations [68].

Together, these studies show that the BMP pathway can have both protective effects, as in $\mathrm{PAH}$, and antagonistic effects, as in atherosclerosis. Although the same major BMPs are involved in both cases, the differing outcomes suggest that additional players, such as the receptors, must have significant roles in determining the outcome. These effects are beginning to be elucidated, for example through the recent studies on the regulator BMPER, but additional work is necessary to truly understand the intracellular mechanisms.

\section{Concluding remarks}

As one of the major signaling pathways in endothelial cells, the BMP pathway is understandably complex, as highlighted by the number of ligands, the diversity of receptors, and the myriad functions and intracellular cascades that it affects. This intricate pathway is essential for endothelial homeostasis and responding to both normal stressors (e.g., laminar shear and oxidative stress) and abnormal stressors (e.g., inflammation, hypoxia, and hypertension). The BMP pathway has been implicated in disorders ranging from CCM and chronic kidney disease to cancer. This diversity exemplifies the need for careful future investigations to determine how the environmental context and specific endothelial populations work together to result in a healthy vasculature. This future work is also crucial for elucidating the specific intracellular signals that are affected in pathologies such as hypertension and atherosclerosis, which will allow for more targeted therapeutic approaches.

\section{Acknowledgments}

We would like to thank Andrea Portbury and Davin Townley-Tilson for critical reading of the manuscript and the NIH (grant \#R01HL061656) for funding support.

\section{glossary}

Angiogenesis

BAECs

Bone morphogenetic protein (BMP)

BMP receptors (BMPRs) the process of generating new blood vessels from existing blood vessels; requires endothelial proliferation, sprouting and migration from existing vessels, and tube formation in the next destination

bovine arterial endothelial cells one of the two major subfamilies of the TGF- $\beta$ super family regulatory growth factor

a family of transmembrane serine/threonine kinases that include type I (BMPR1A) and type II receptor (BMPR1B, BMPR2) 


\section{Cerebral cavernous malformation (CCM)}

Endoglin

Endothelial nitric oxide synthase (eNOS)

Extracellular signalregulated kinase (ERK)

HAECs

Hereditary

hemorrhagic

telangiectasia (HHT)

HPAECS

HUVECS

Laminar shear stress

MGP

Mitogen-activated protein kinase (MAPK)

Oscillatory shear stress

PAECs

Pulmonary arterial hypertension (PAH)

Sprouting

Vascular endothelial growth factor (VEGF) characterized by having a collection of large, irregularly shaped veins that typically have thin walls and no supporting tissue, making them prone to leaking

a type I glycoprotein that is restricted to the endothelium; also known as a type III BMP receptor

a calcium-dependent nitric oxide synthase isoform that is restricted to endothelial cells

one branch of the MAP kinase pathway

human arterial endothelial cells

characterized by permanently dilated vessels, predominantly

in the skin and mucosa, and gastrointestinal bleeding

human pulmonary artery endothelial cells

human umbilical vein endothelial cells

the stress caused by steady, typically high-velocity blood flow matrix Gla protein

a superfamily of protein-serine-threonine kinases activated by numerous stimuli

the stress caused by turbulent, non-laminar blood flow

pulmonary artery endothelial cells

increased vascular resistance within the pulmonary circulation

an invasive endothelial behavior that involves identifying a single leading cell that will migrate away from an existing vessel and bring its neighboring cells to form new vessels; the leading tip cell signals via the Notch pathway to inhibit the following (stalk) neighbor cells from also becoming tip cells a family of growth factors that specifically stimulates vascular growth and angiogenesis

\section{References}

1. Cai J, et al. BMP signaling in vascular diseases. FEBS Lett. 2012; 586:1993-2002. [PubMed: 22710160]

2. Wiley DM, Jin SW. Bone Morphogenetic Protein functions as a context-dependent angiogenic cue in vertebrates. Semin Cell Dev Biol. 2011

3. Bandyopadhyay A, et al. BMP signaling in development and diseases: a pharmacological perspective. Biochemical pharmacology. 2013; 85:857-864. [PubMed: 23333766] 
4. Maddaluno L, et al. EndMT contributes to the onset and progression of cerebral cavernous malformations. Nature. 2013; 498:492-496. [PubMed: 23748444]

5. Pi X, et al. Sequential roles for myosin-X in BMP6-dependent filopodial extension, migration, and activation of BMP receptors. The Journal of cell biology. 2007; 179:1569-1582. [PubMed: 18158328]

6. Ciumas M, et al. Bone morphogenetic proteins protect pulmonary microvascular endothelial cells from apoptosis by upregulating alpha-B-crystallin. Arterioscler Thromb Vasc Biol. 2013; 33:25772584. [PubMed: 24072698]

7. David L, et al. Emerging role of bone morphogenetic proteins in angiogenesis. Cytokine Growth Factor Rev. 2009; 20:203-212. [PubMed: 19502096]

8. Yao Y, et al. Heat shock protein 70 enhances vascular bone morphogenetic protein- 4 signaling by binding matrix Gla protein. Circ Res. 2009; 105:575-584. [PubMed: 19661459]

9. Yao Y, et al. Matrix Gla protein deficiency causes arteriovenous malformations in mice. J Clin Invest. 2011; 121:2993-3004. [PubMed: 21765215]

10. Suzuki Y, et al. BMP-9 induces proliferation of multiple types of endothelial cells in vitro and in vivo. J Cell Sci. 2010; 123:1684-1692. [PubMed: 20406889]

11. Brown MA, et al. Crystal structure of BMP-9 and functional interactions with pro-region and receptors. J Biol Chem. 2005; 280:25111-25118. [PubMed: 15851468]

12. Orlova VV, et al. Controlling angiogenesis by two unique TGF-beta type I receptor signaling pathways. Histology and histopathology. 2011; 26:1219-1230. [PubMed: 21751154]

13. David L, et al. Identification of BMP9 and BMP10 as functional activators of the orphan activin receptor-like kinase 1 (ALK1) in endothelial cells. Blood. 2007; 109:1953-1961. [PubMed: 17068149]

14. David L, et al. Bone morphogenetic protein-9 is a circulating vascular quiescence factor. Circ Res. 2008; 102:914-922. [PubMed: 18309101]

15. Scharpfenecker M, et al. BMP-9 signals via ALK1 and inhibits bFGF-induced endothelial cell proliferation and VEGF-stimulated angiogenesis. J Cell Sci. 2007; 120:964-972. [PubMed: 17311849]

16. Townson SA, et al. Specificity and structure of a high affinity activin receptor-like kinase 1 (ALK1) signaling complex. J Biol Chem. 2012; 287:27313-27325. [PubMed: 22718755]

17. Nolan-Stevaux O, et al. Endoglin requirement for BMP9 signaling in endothelial cells reveals new mechanism of action for selective anti-endoglin antibodies. PLoS One. 2012; 7:e50920. [PubMed: 23300529]

18. Park JE, et al. BMP-9 induced endothelial cell tubule formation and inhibition of migration involves Smad1 driven endothelin-1 production. PLoS One. 2012; 7:e30075. [PubMed: 22299030]

19. Upton PD, et al. Bone morphogenetic protein (BMP) and activin type II receptors balance BMP9 signals mediated by activin receptor-like kinase-1 in human pulmonary artery endothelial cells. J Biol Chem. 2009; 284:15794-15804. [PubMed: 19366699]

20. Finkenzeller G, et al. Effects of bone morphogenetic protein 2 on human umbilical vein endothelial cells. Microvasc Res. 2012; 84:81-85. [PubMed: 22487440]

21. Upton PD, et al. Functional characterization of bone morphogenetic protein binding sites and Smad1/5 activation in human vascular cells. Mol Pharmacol. 2008; 73:539-552. [PubMed: 17989347]

22. Pi X, et al. LRP1-dependent endocytic mechanism governs the signaling output of the bmp system in endothelial cells and in angiogenesis. Circ Res. 2012; 111:564-574. [PubMed: 22777006]

23. Tian XY, et al. Bone morphogenic protein-4 induces endothelial cell apoptosis through oxidative stress-dependent p38MAPK and JNK pathway. J Mol Cell Cardiol. 2012; 52:237-244. [PubMed: 22064324]

24. Wong WT, et al. Bone morphogenic protein-4 impairs endothelial function through oxidative stress-dependent cyclooxygenase-2 upregulation: implications on hypertension. Circulation Research. 2010; 107:984-991. [PubMed: 20724703]

25. Yu PB, et al. Bone morphogenetic protein (BMP) type II receptor deletion reveals BMP ligandspecific gain of signaling in pulmonary artery smooth muscle cells. J Biol Chem. 2005; 280:24443-24450. [PubMed: 15883158] 
26. de Jesus Perez VA, et al. Bone morphogenetic protein 2 induces pulmonary angiogenesis via Wntbeta-catenin and Wnt-RhoA-Rac1 pathways. The Journal of cell biology. 2009; 184:83-99. [PubMed: 19139264]

27. Tian H, et al. Endoglin mediates fibronectin/alpha5beta1 integrin and TGF-beta pathway crosstalk in endothelial cells. EMBO J. 2012; 31:3885-3900. [PubMed: 22940691]

28. Kelley R, et al. A concentration-dependent endocytic trap and sink mechanism converts Bmper from an activator to an inhibitor of Bmp signaling. J Cell Biol. 2009; 184:597-609. [PubMed: 19221194]

29. Pi X, et al. Bmper inhibits endothelial expression of inflammatory adhesion molecules and protects against atherosclerosis. Arterioscler Thromb Vasc Biol. 2012; 32:2214-2222. [PubMed: 22772758]

30. Moreno-Miralles I, et al. Bone morphogenetic protein endothelial cell precursor-derived regulator regulates retinal angiogenesis in vivo in a mouse model of oxygen-induced retinopathy. Arteriosclerosis, thrombosis, and vascular biology. 2011; 31:2216-2222.

31. Moser M, et al. BMPER is a conserved regulator of hematopoietic and vascular development in zebrafish. J Mol Cell Cardiol. 2007; 43:243-253. [PubMed: 17618647]

32. Teichert-Kuliszewska $\mathrm{K}$, et al. Bone morphogenetic protein receptor-2 signaling promotes pulmonary arterial endothelial cell survival: implications for loss-of-function mutations in the pathogenesis of pulmonary hypertension. Circ Res. 2006; 98:209-217. [PubMed: 16357305]

33. Xu J, et al. Over-expression of BMP4 inhibits experimental choroidal neovascularization by modulating VEGF and MMP-9. Angiogenesis. 2012; 15:213-227. [PubMed: 22392094]

34. Wiley DM, et al. Distinct signalling pathways regulate sprouting angiogenesis from the dorsal aorta and the axial vein. Nature cell biology. 2011; 13:686-692.

35. Kim JD, et al. Context-dependent proangiogenic function of bone morphogenetic protein signaling is mediated by disabled homolog 2. Dev Cell. 2012; 23:441-448. [PubMed: 22898784]

36. Osada M, et al. Platelet activation receptor CLEC-2 regulates blood/lymphatic vessel separation by inhibiting proliferation, migration, and tube formation of lymphatic endothelial cells. J Biol Chem. 2012; 287:22241-22252. [PubMed: 22556408]

37. Ricard N, et al. BMP9 and BMP10 are critical for postnatal retinal vascular remodeling. Blood. 2012; 119:6162-6171. [PubMed: 22566602]

38. Larrivee B, et al. ALK1 signaling inhibits angiogenesis by cooperating with the Notch pathway. Dev Cell. 2012; 22:489-500. [PubMed: 22421041]

39. Somekawa S, et al. Tmem100, an ALK1 receptor signaling-dependent gene essential for arterial endothelium differentiation and vascular morphogenesis. Proc Natl Acad Sci U S A. 2012; 109:12064-12069. [PubMed: 22783020]

40. Moya IM, et al. Stalk cell phenotype depends on integration of Notch and Smad1/5 signaling cascades. Dev Cell. 2012; 22:501-514. [PubMed: 22364862]

41. Roca C, Adams RH. Regulation of vascular morphogenesis by Notch signaling. Genes Dev. 2007; 21:2511-2524. [PubMed: 17938237]

42. Yao Y, et al. Crossveinless 2 regulates bone morphogenetic protein 9 in human and mouse vascular endothelium. Blood. 2012; 119:5037-5047. [PubMed: 22474252]

43. Poirier O, et al. Inhibition of apelin expression by BMP signaling in endothelial cells. Am J Physiol Cell Physiol. 2012; 303:C1139-C1145. [PubMed: 22895257]

44. Tian F, et al. Endothelial cells are activated during hypoxia via endoglin/ALK-1/SMAD1/5 signaling in vivo and in vitro. Biochem Biophys Res Commun. 2010; 392:283-288. [PubMed: 20060813]

45. Guillot N, et al. Accelerated reendothelialization, increased neovascularization and erythrocyte extravasation after arterial injury in BAMBI ${ }^{\mathrm{TM}} / \mathrm{TM}$ mice. PLoS One. 2013; 8:e58550. [PubMed: 23469285]

46. Kim YW, Byzova TV. Oxidative stress in angiogenesis and vascular disease. Blood. 2014; 123:625-631. [PubMed: 24300855]

47. Zhang Y, et al. Inhibition of bone morphogenic protein 4 restores endothelial function in $\mathrm{db} / \mathrm{db}$ diabetic mice. Arterioscler Thromb Vasc Biol. 2014; 34:152-159. [PubMed: 24202302] 
48. Brook RD, et al. Beyond medications and diet: alternative approaches to lowering blood pressure: a scientific statement from the american heart association. Hypertension. 2013; 61:1360-1383. [PubMed: 23608661]

49. Gangopahyay A, et al. Bone morphogenetic protein receptor II is a novel mediator of endothelial nitric-oxide synthase activation. J Biol Chem. 2011; 286:33134-33140. [PubMed: 21808054]

50. Anderson L, et al. Bmp2 and Bmp4 exert opposing effects in hypoxic pulmonary hypertension. Am J Physiol Regul Integr Comp Physiol. 2010; 298:R833-R842. [PubMed: 20042692]

51. Star GP, et al. Bone morphogenic protein- 9 stimulates endothelin-1 release from human pulmonary microvascular endothelial cells: a potential mechanism for elevated ET-1 levels in pulmonary arterial hypertension. Microvasc Res. 2010; 80:349-354. [PubMed: 20594999]

52. Ma L, Chung WK. The genetic basis of pulmonary arterial hypertension. Human genetics. 2014

53. Alastalo TP, et al. Disruption of PPARgamma/beta-catenin-mediated regulation of apelin impairs BMP-induced mouse and human pulmonary arterial EC survival. J Clin Invest. 2011; 121:37353746. [PubMed: 21821917]

54. Spiekerkoetter E, et al. FK506 activates BMPR2, rescues endothelial dysfunction, and reverses pulmonary hypertension. J Clin Invest. 2013; 123:3600-3613. [PubMed: 23867624]

55. Choi EJ, et al. Enhanced responses to angiogenic cues underlie the pathogenesis of hereditary hemorrhagic telangiectasia 2. PLoS One. 2013; 8:e63138. [PubMed: 23675457]

56. Yao Y, et al. Reducing Jagged 1 and 2 levels prevents cerebral arteriovenous malformations in matrix Gla protein deficiency. Proc Natl Acad Sci U S A. 2013; 110:19071-19076. [PubMed: 24191040]

57. Go AS, et al. Heart disease and stroke statistics--2014 update: a report from the american heart association. Circulation. 2014; 129:e28-e292. [PubMed: 24352519]

58. Yao Y, et al. Inhibition of bone morphogenetic proteins protects against atherosclerosis and vascular calcification. Circ Res. 2010; 107:485-494. [PubMed: 20576934]

59. Wentzel JJ, et al. Endothelial shear stress in the evolution of coronary atherosclerotic plaque and vascular remodelling: current understanding and remaining questions. Cardiovasc Res. 2012; 96:234-243. [PubMed: 22752349]

60. Csiszar A, et al. Hemodynamic forces, vascular oxidative stress, and regulation of BMP-2/4 expression. Antioxidants \& redox signaling. 2009; 11:1683-1697. [PubMed: 19320562]

61. Sorescu GP, et al. Bone morphogenic protein 4 produced in endothelial cells by oscillatory shear stress induces monocyte adhesion by stimulating reactive oxygen species production from a nox 1 based NADPH oxidase. Circ Res. 2004; 95:773-779. [PubMed: 15388638]

62. Zhou J, et al. BMP receptor-integrin interaction mediates responses of vascular endothelial Smad1/5 and proliferation to disturbed flow. Journal of thrombosis and haemostasis : JTH. 2013; 11:741-755. [PubMed: 23387849]

63. Ross TD, et al. Integrins in mechanotransduction. Curr Opin Cell Biol. 2013; 25:613-618. [PubMed: 23797029]

64. Albrecht EW, et al. Protective role of endothelial nitric oxide synthase. The Journal of pathology. 2003; 199:8-17. [PubMed: 12474221]

65. Helbing T, et al. BMP activity controlled by BMPER regulates the proinflammatory phenotype of endothelium. Blood. 2011; 118:5040-5049. [PubMed: 21900199]

66. Helbing T, et al. BMPER is upregulated by statins and modulates endothelial inflammation by intercellular adhesion molecule-1. Arterioscler Thromb Vasc Biol. 2010; 30:554-560. [PubMed: 20042706]

67. Kim CW, et al. Anti-inflammatory and antiatherogenic role of BMP receptor II in endothelial cells. Arterioscler Thromb Vasc Biol. 2013; 33:1350-1359. [PubMed: 23559633]

68. Derwall M, et al. Inhibition of bone morphogenetic protein signaling reduces vascular calcification and atherosclerosis. Arterioscler Thromb Vasc Biol. 2012; 32:613-622. [PubMed: 22223731]

69. Su X, et al. Oxidized low density lipoprotein induces bone morphogenetic protein-2 in coronary artery endothelial cells via Toll-like receptors 2 and 4. J Biol Chem. 2011; 286:12213-12220. [PubMed: 21325271] 
70. Staton CA, et al. A critical analysis of current in vitro and in vivo angiogenesis assays. Int J Exp Pathol. 2009; 90:195-221. [PubMed: 19563606]

71. Stahl A, et al. The mouse retina as an angiogenesis model. Invest Ophthalmol Vis Sci. 2010; 51:2813-2826. [PubMed: 20484600] 


\section{Text box 1: Models of angiogenesis}

Numerous in vivo models are used to study angiogenesis. Three of these models include tumor xenografts, Matrigel plugs, and retinopathy models. Herein, these models will be briefly described, including benefits and disadvantages.

\section{Xenograft model}

In the xenograft model, human tumor cells are injected into a host mouse or rat. These cells can then be followed as they graft into the host animal and form a tumor. This model is incredibly easy for investigating tumor growth, which can be measured based on size and weight. Further, the overall vascularization of the tumor can be grossly assessed by excision, followed by more detailed histology if desired. An additional benefit of this model is that novel test compounds can be evaluated for whether they inhibit vascular recruitment to the growing tumor or whether they affect the endothelial cells after connecting to the host vasculature. However, not all injected tumor cells will successfully form a tumor [70].

\section{Matrigel plug model}

Similarly, in the Matrigel plug model, the commercially available extracellular matrix product Matrigel is mixed with a chemical or cell type of interest and then injected into a host animal. One advantage of Matrigel is that it is liquid at $4^{\circ} \mathrm{C}$, allowing it to be mixed with other reagents, and then forms a solid gel at $37^{\circ} \mathrm{C}$. Thus, the injected Matrigel forms a "plug" that can be invaginated like a tumor. However, to visualize the vasculature, the plug must be excised and subjected to histological analysis [70].

\section{Retinopathy model}

The retinal vascular plexus forms following a stereotypical radial pattern, which makes abnormal developmental patterns easy to identify. Premature infants often require hyperoxic environments, which lead to vascular obliteration in the retina until the infant is returned to a normoxic - now relatively hypoxic - environment. Although the relatively hypoxic environment stimulates neovascularization, these changes can lead to prematurity of retinopathy, a major cause of acquired blindness. To study this process in mouse, mouse litters can be maintained under hyperoxic conditions, which cause the retinal vasculature to regress. When the pups are returned to a normoxic environment, this environment is again relatively hypoxic, and vascular growth is stimulated. Using this model, genetic mouse models and applied agents can be tested for their effects on preventing vascular regression or affecting the rate of neovascularization. One caveat is that the retinal dissections are particularly delicate [71]. 


\section{Highlights}

- In addition to BMP2 and BMP4, BMP9 plays crucial, context-specific roles in the endothelium.

- The recently coined "type III" co-receptors interact with the type I and type II receptors and typically lead to endocytotic recycling of the BMP/BMP receptor complex.

- BMPs activate different intracellular signaling cascades that plays key roles in hypertension, vascular disorders, and atherosclerosis. 
A

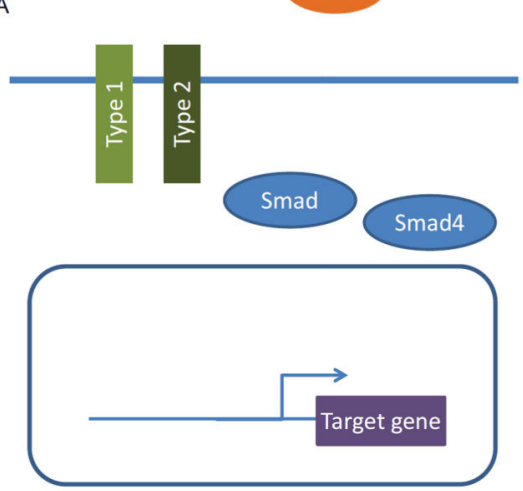

D

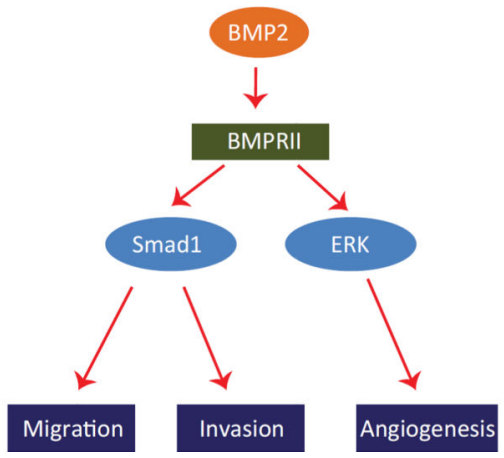

B

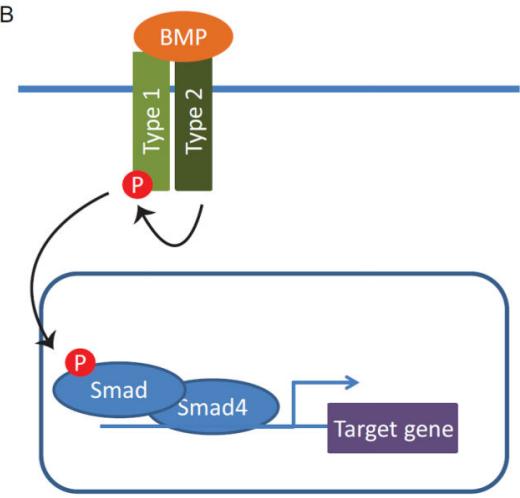

$\mathrm{E}$

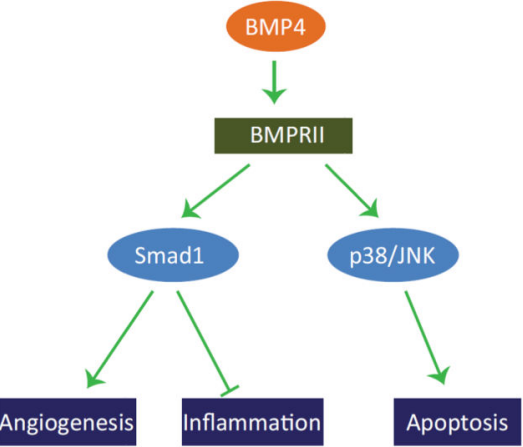

C

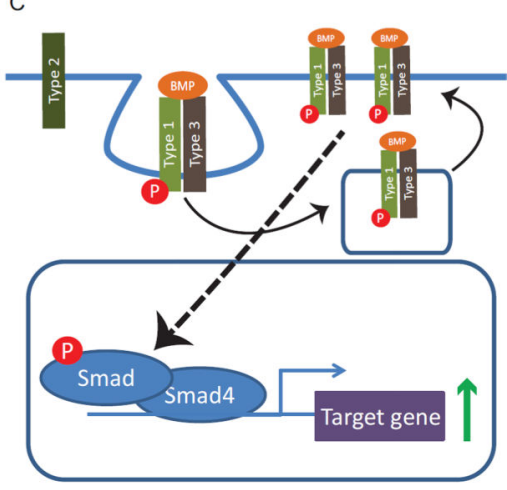

$\mathrm{F}$

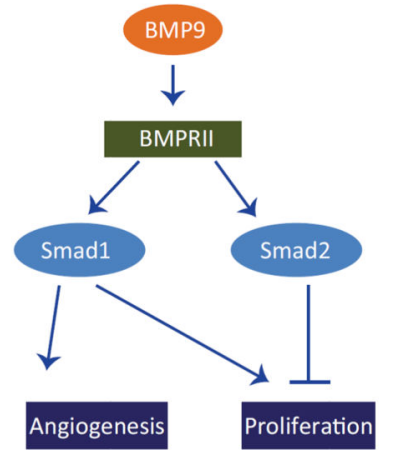

Figure 1. An overview of the canonical BMP pathway

(A) In the absence of BMP binding (top panel), BMP receptors type I and II do not associate, and the Smad transcription factors remain in the cytoplasm. (B) BMP typically binds to the type II receptor (bottom panel), which allows type I and II receptors dimerize and the type II receptor to phosphorylate the type I receptor. However, in some cases, the BMP ligand has a higher affinity for the type I receptor, and this binding will then induce dimerization and subsequent phosphorylation. This phosphorylation of the type I receptor leads to the phosphorylation of downstream Smads, canonically Smads 1/5/8, which then associate with the co-Smad, Smad4, and translocate into the nucleus. The Smad complex will bind to BMP response elements and induce the transcription of downstream targets. (C) The recently discovered type III receptors can compete with the type II receptors and form a complex with the type I receptors. This association leads to endocytosis-mediated recycling of the ligand-receptor complex to the membrane, which amplifies the BMP signaling pathway. (D-F) Although the canonical pathway is seemingly straightforward, a single ligand can have numerous effects and outcomes in endothelial cells. Depicted here are the effects of BMP2 (D), BMP4 (E), and BMP9 (F) specifically via BMPRII. Each ligand can signal through the canonical Smad1 or through a different intracellular cascade. Further, even a single ligand can have opposing effects on a single behavior (e.g., BMP9 and proliferation) via the intracellular signaling cascade that is activated. For simplicity, type I and type III receptors, if identified, as well as downstream targets have been omitted. 


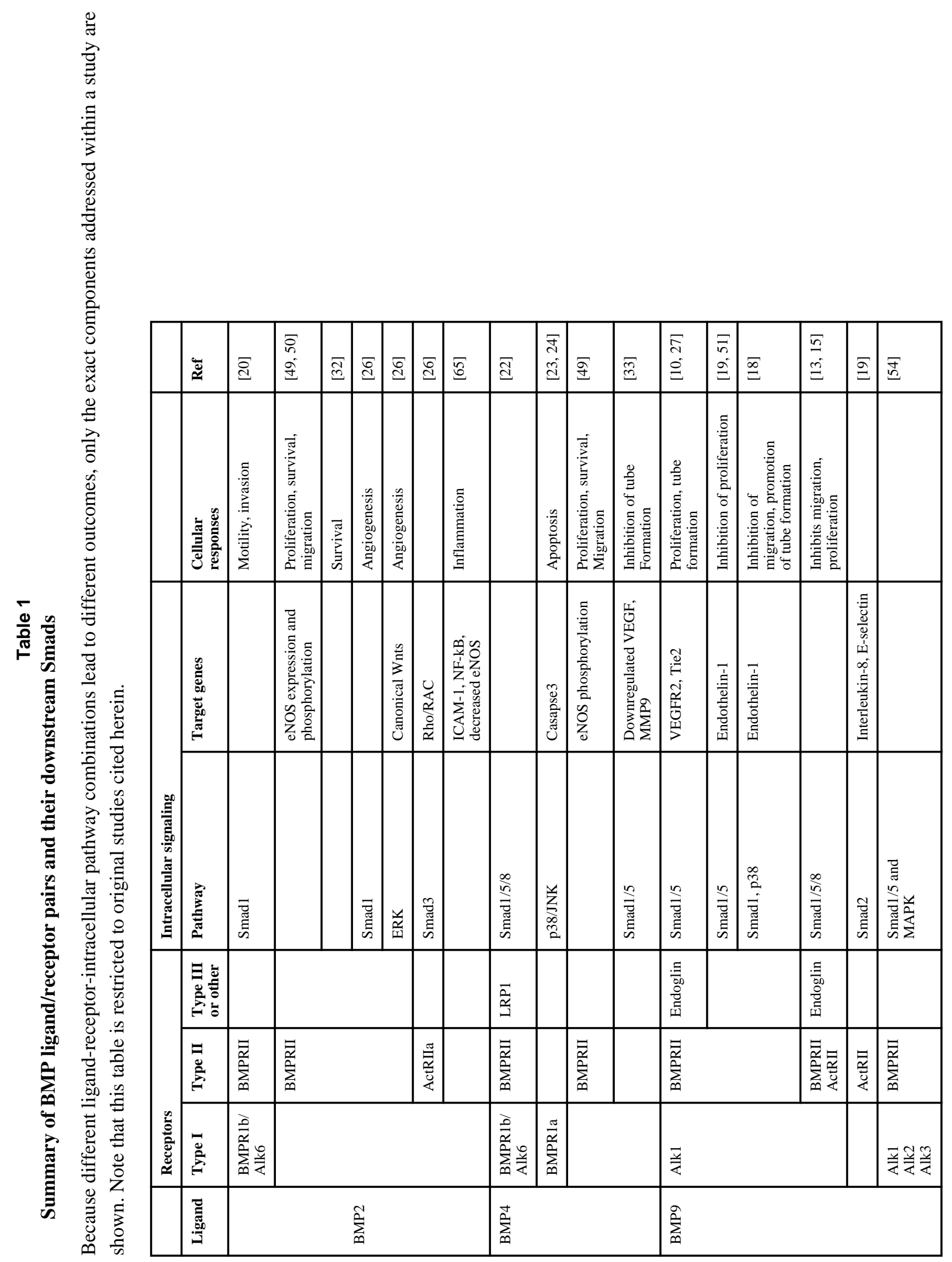




\begin{tabular}{|c|c|c|c|c|c|c|c|c|}
\hline & Æ & 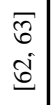 & $\Phi$ & $\bar{d}$ & 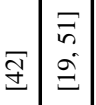 & $\bar{\Omega}$ & $\bar{\Xi}$ & E్ \\
\hline & 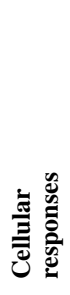 & 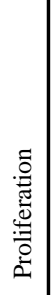 & 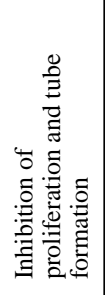 & 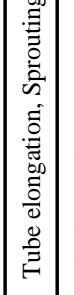 & 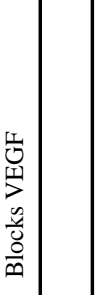 & 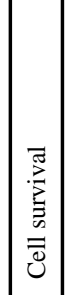 & 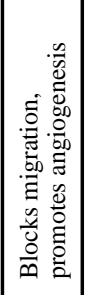 & 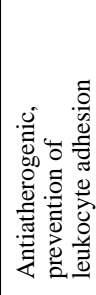 \\
\hline & 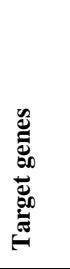 & & & 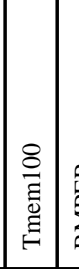 & 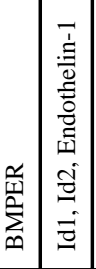 & $\mid$ & 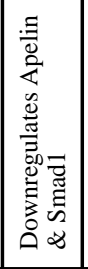 & 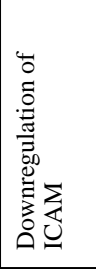 \\
\hline 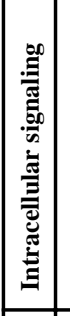 & 咅 & 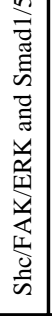 & & & 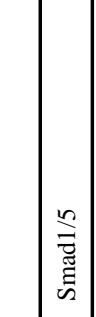 & & & 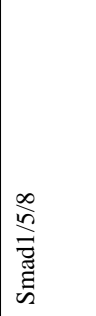 \\
\hline & 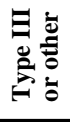 & & & & & 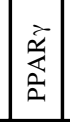 & & \\
\hline & 总 & 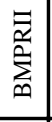 & & & & 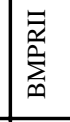 & & \\
\hline 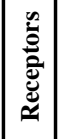 & 彦 & 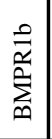 & $\overrightarrow{\underline{z}}$ & & & & & \\
\hline . & 惹 & & & & & & & \\
\hline
\end{tabular}

Trends Endocrinol Metab. Author manuscript; available in PMC 2015 September 01. 